\title{
DIAGNOSTIC CRITERIA OF TEMPORO-MANDIBULAR JOINT OSTEOARTHRITIS ON COMPUTED TOMOGRAPHY
}

\author{
Khaydarova Gozal Bagiddinovna \\ Assistant of Oncology and radiology department of Tashkent Medical \\ Academy (e-mail strelets-1985@bk.ru)
}

Boymuradov Shuxrat Abdujalilovich

Vice-rector of scientific work of Tashkent Medical Academy

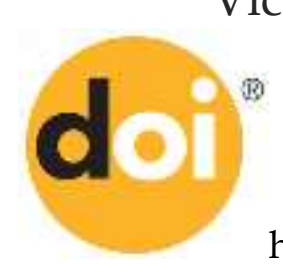

http:/ / dx.doi.org/10.26739/2573-5616-2018-1-2-9

Abstract: This article is devoted to applications of computed tomography (CT) in diagnosis of inflammatory and degenerative disease of temporomandibular joint (TMJ). CT is highly informative method for visualization of disease of temporomandibular joint. Finally, sections on other aspects of CT research related to the TMJ, clinical decision-making and concluding remarks are added. However, it should be emphasized that the diagnostic information obtained is limited to the morphology of the osseous joint components, cortical bone integrity and subcortical bone destruction/production. Author of this research studied the frequency of radiological signs in different age group. In addition, there were studied frequency of isolated, combination types, and more and less localization sites of radiological patterns of inflammatory and degenerative changes of temporo-mandibular joint. 
Keywords: Degenerative joint disease, temporomandibular joint osteoarthritis; diagnostic imaging; temporomandibular disorders; joint pain; masticatory muscle pain.

Osteoarthritis (OA) of the temporomandibular joint (TMJ) is defined as a degenerative joint condition, characterized by involvement of the joint tissue and concomitant remodeling of the main subchondral bone [1]. 15\% of the world's population suffers from osteoarthritis of the TMJ; clinical signs of osteoarthritis of the TMJ are manifested in $8-16 \%$ of the general population [4]. Osteoarthritis of the TMJ can be unilateral, bilateral, and more common in women.

Osteoarthritis is associated more with the inflammatory process. Risk factors include age, heredity, traumas, disorders of joint structures and muscles, systemic lesions (systemic osteoarthritis, infections, idiopathic degenerative processes, congenital and acquired pathologies)[8]. Mechanical and metabolic factors lead to early destruction of cartilage. Inflammatory mediators, such as cytokines and chemokines, activate the degrading factor of the cartilage and produce matrix-metalloproteinases and prostaglandin E. This process extends to the deeper layer of cartilage, forming marginal bone erosion [3].

Cardinal signs of OA of the TMJ are revealed by clinical and radiographic methods [4]. Clinical features include joint stiffness, joint pain during mouth opening and lateral excursions, often accompanied by a hard sound or crepitus $[4,5]$.

X-ray signs of the disease are erosion of the cortical bone, flattening of joint surfaces with productive changes in bones, such as sclerosis and osteophytes [6]. These signs of the TMJ OA are present different stages of the disease process.

Erosive lesions, subchondral bone changes and narrowing of the articular space indicate acute or early changes, whereas sclerosis, flattening, subchondral cyst and osteophyte may indicate late changes in the TMJ [7]. CT is often used to determine the early changes of the articular disc and study the structure of the joint in different planes, including with 3D reconstruction. Despite patient irradiation, CT remains the method of choice, since it allows to determine early and late signs of TMJ disease before and after treatment of the patient. CT is especially informative for determining bone changes, such as erosion, trauma, post-surgical deformities, as well as changes in the Social science and humanities 
temporal bone, the condylo-coronoidal angle that changes in the pathology of the TMJ. The research was carried out with the opening and closing of the mouth to determine the condition of the joint disc (thickness of sections from 1 to $2.5 \mathrm{~mm})$.

In the present work, the frequency of occurrence and features of various CT signs were studied in patients with OA of the TMJ in various age groups.

\section{Material and methods}

Studies were carry out in 56 patients aged 50 to 80 years, 36 women and 20 men. All of them were examined for temporomandibular dysfunction.

Tenderness in the TMJ and the mandible was assessed by bilateral palpation of the preauric region when the mouth was opened and closed. Stiffness in the periarticular muscles was checked by palpation of each muscle.

The presence of a crack in the area of the TMJ was verified by palpation of the sides of the joint near the preauric region or auscultation of the preauric region, with the patient doing three openings and lateral movements in the joint.

Clinical criteria were formulated using the standard Okeson questionnaire, which includes: (1) stiffness present in the prearicular region, (2) stiffness in the masticatory muscles, (3) restriction or deflection in the range of motion of the mandible (restriction of mouth opening to $30 \%$ ) (4) click, or pop-up, or crepitus [8].

Multispiral computed tomography of the TMJ was performed on a 6 cutter (SIEMENS SOMATOM EMOTION, $125 \mathrm{kV}, 500 \mathrm{~mA}$ ), thin sections (thickness from 1 to $2.5 \mathrm{~mm}$ ). Postprocessing analysis included obtaining coronal-oblique sections (parallel to the axis of the condyles of the mandible), sagittal-oblique sections in bone and soft tissue regimes. Also used $3 \mathrm{D}$ reconstruction.

\section{Results}

CT scan of the control group showed that the normal cortical bone of the mandible condyle has smooth contours. The condyles look wider in the coronary planes. The anterior and posterior horns of the articular disc are of a higher density than the surrounding soft tissues. Bilaminar and median zone of the disk on CT scans without contrast was not visualized.

The results of the study showed that of the 112 joints evaluated in 56 patients, bone changes were present in $88(78 \%)$ joints, either in condylar or 
in the condylar fossa (Fig. 1) in the articulation. They could reveal their combination. Unilateral lesion was noted in 24 patients, bilateral lesion in 32 patients.

Osteoarthritis of the TMJ was manifested by the following changes in CT imaging:

1. Narrowing of the joint space - reduction of the space between the condyles and the glenoid fossa in all directions (anterior, upper and posterior). A narrowing (Fig. 1A.) was considered a reduction in the width of the slit of less than $1.5 \mathrm{~mm}$, normal from $1.5 \mathrm{~mm}$ to $4.0 \mathrm{~mm}$, expanded more than $4.0 \mathrm{~mm}$.

2. Erosion - interruption or absence of cortical bone (Fig. 2)

3. Osteophytis-marginal outgrowths of bones (Fig. 3.)

4. Sclerosis - increasing the density of the cortical or subchondral bone

5. Subchondral cyst - single or multiple rounded subchondral lesions up to $2 \mathrm{~mm}$ in size, having sclerotic margins (1B.)

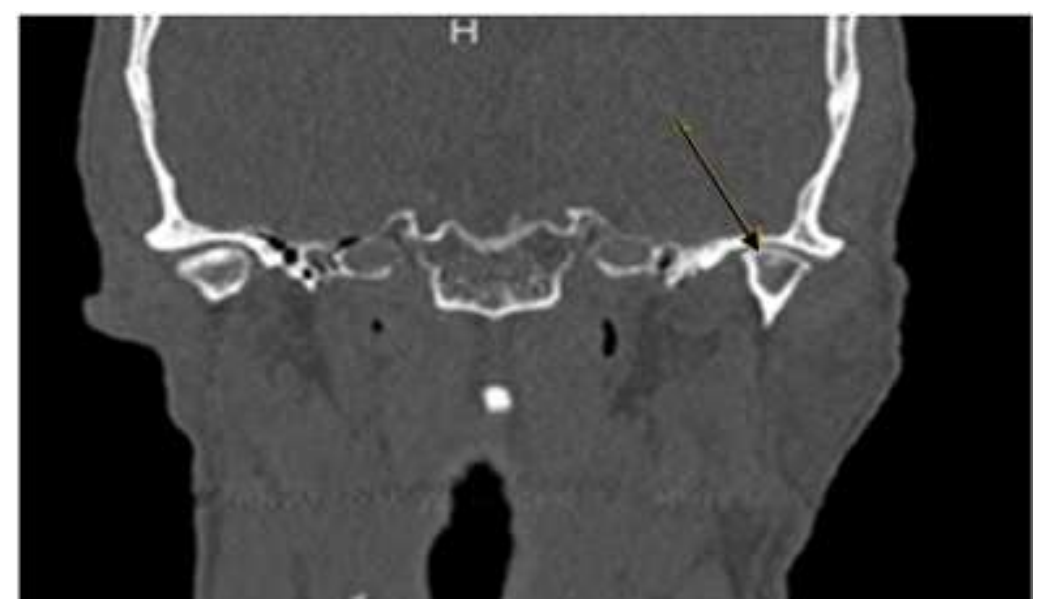

Fig-1A. CT of patient V., 60 years old. Coronal section of the TMJ. The arrow indicates the narrowing of the joint space and moderate mushroom deformation of the head of the condylar process of the mandible to the left. 


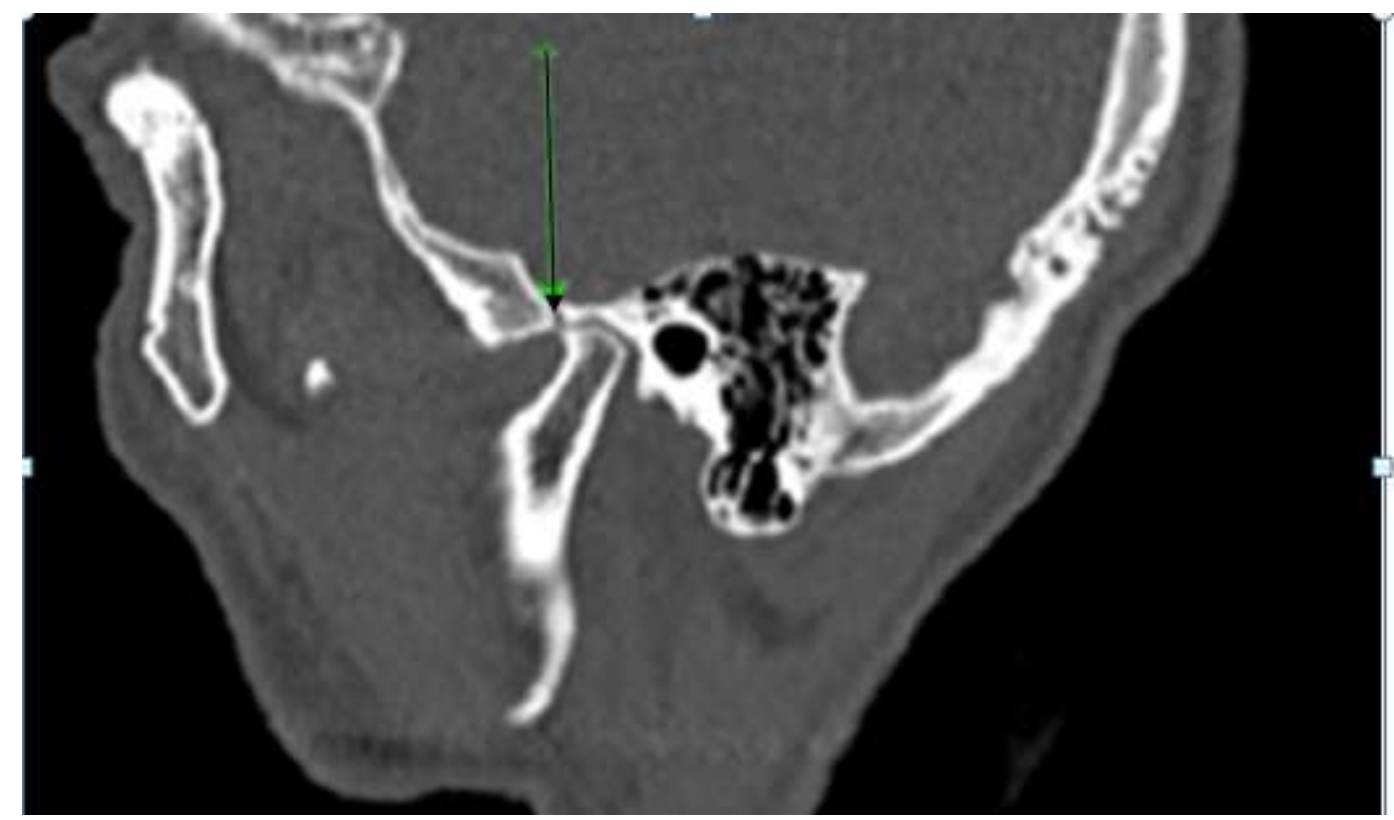

Fig-1B. CT of the same patient, sagittal section of the TMJ on the left. The arrow indicates a small subchondral cyst in the anterior part of the temporal fossa of the temporal bone.

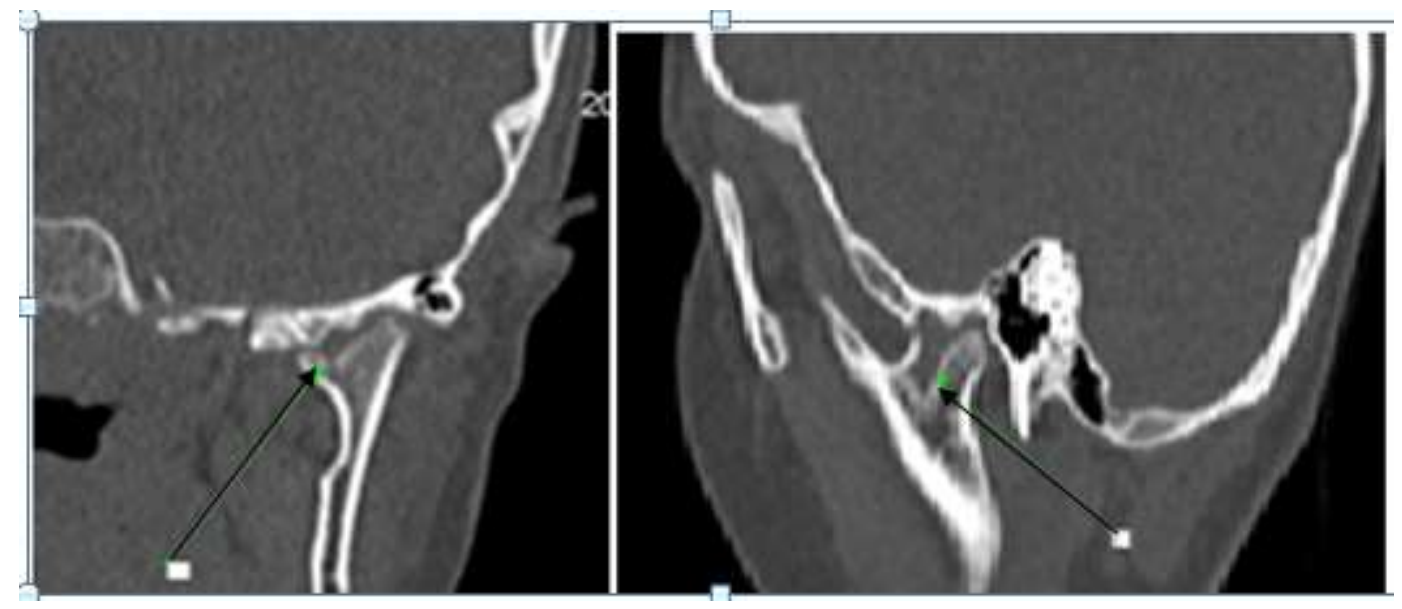

Pic-2. CT of patient A., 61 years old. (A-coronal section, B-sagittal section). Expressed erosive changes along the inner-anterior surface of the head of the condyle of the mandible and destructive changes in the joint fossa of the temporal bone on the left. 


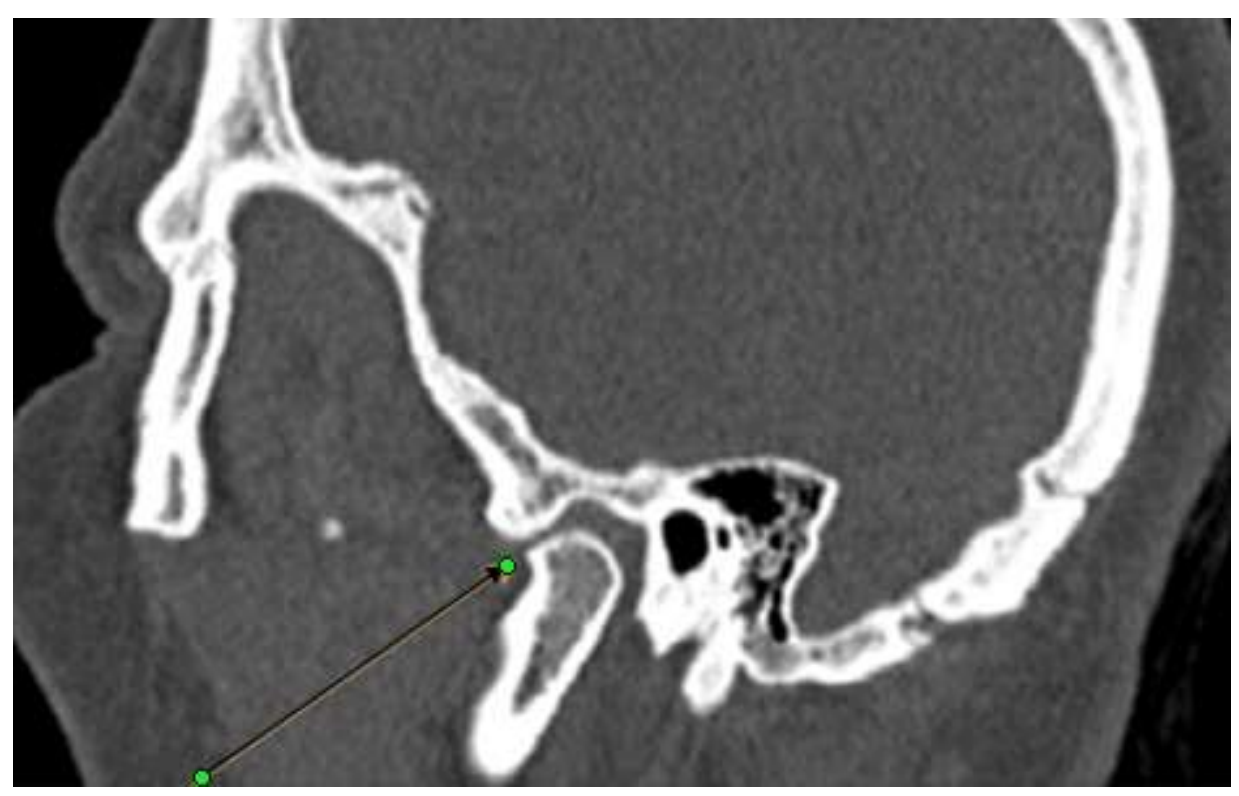

Pic-3. CT of the patient L. 65 years old. Sagittal section. A small marginal aggravation due to bone growth on the anterior contour of the head of the condyle of the mandible.

In most observations on CT scans, the OA of the TMJ was manifested by a combination of different characters, i.e. narrowing of the joint gap could be combined with marginal bone erosions, osteophytes, subchondral sclerosis, etc.

Osteoarthritis was considered obvious if even one of the five abovementioned joint changes was detected. This occurred only in 8 of the 88 affected joints, the remaining joints visualized a combination of various signs of damage to the joint structures.

Figure- 1 graphically shows the frequency of occurrence of various CT signs of the OA of the TMJ. 


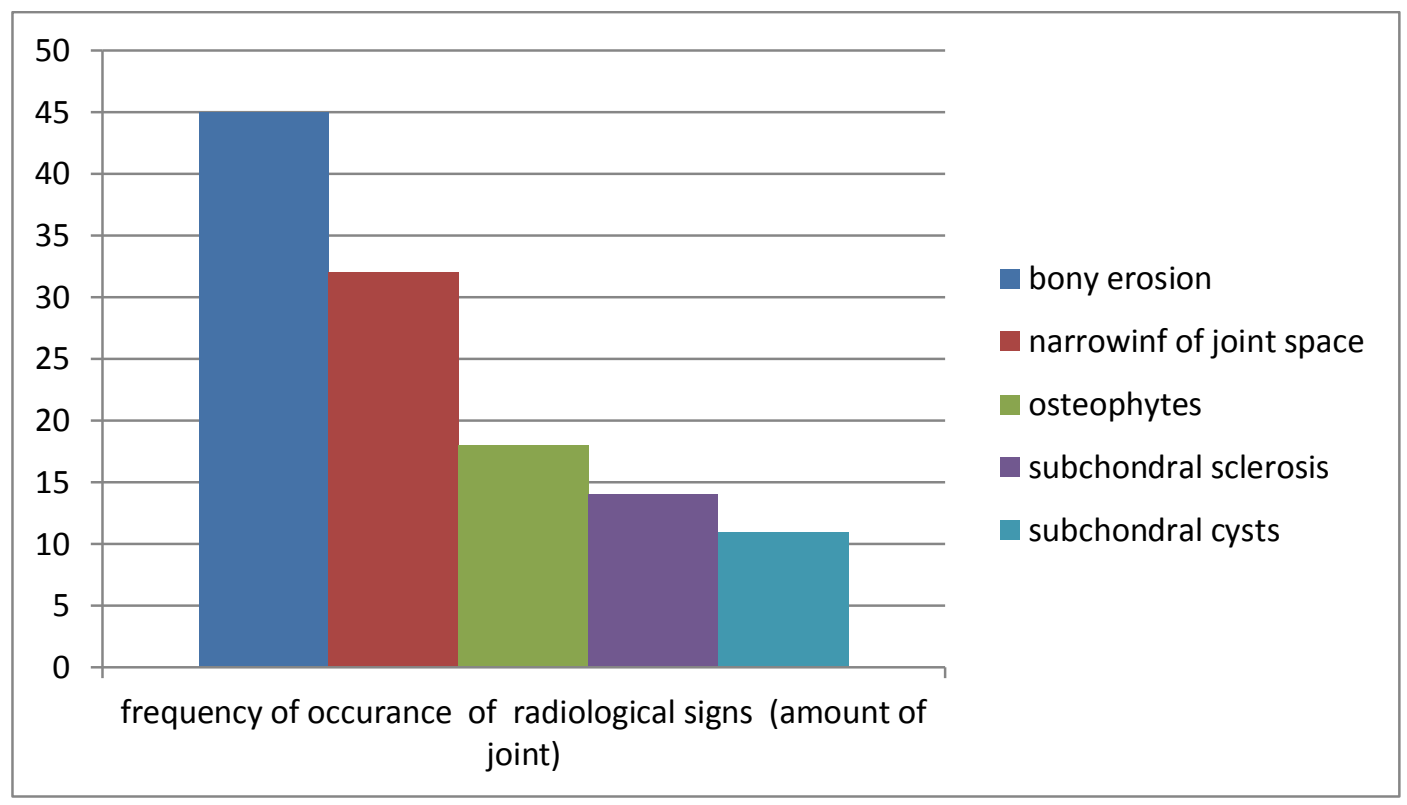

As can be seen from the diagram above, bone erosive changes were most often detected, which are noted in 45 joints. The narrowing of the joint space was revealed in 32 , subchondral cysts in 11 , osteophytes in 18 , sclerosis in 14 joints.

Changes in the head of the condylar process were noted more often than in the joint fossa.

Table 1.

\begin{tabular}{|l|l|c|c|c|}
\hline Radiological signs & $\begin{array}{c}\text { Frequency of } \\
\text { occurance of } \\
\text { radiological } \\
\text { signs }\end{array}$ & $\begin{array}{c}(\%) \\
\text { Age } \\
(\mathrm{M} \pm \mathrm{m})\end{array}$ \\
\hline & Single sign & 8 & 9 & $54 \pm 4$ \\
\hline & The presence of 2 signs & 36 & 41 & $61 \pm 4$ \\
\hline & The presence of 3 signs & 31 & 35 & $68 \pm 3$ \\
\hline & The presence of 4 signs & 13 & 15 & $73 \pm 3$ \\
\hline
\end{tabular}

According to the $\mathrm{CT}$ scan, the combination of various radiological signs of the disease predominated in patients with OA of the TMJ (Table 1). A combination of 2-3 different symptoms (narrowing of the joint space, erosion and osteophytes) was more common.

In the past stages, a combination of erosions and osteophytes with subchondral sclerosis, cysts and false widening of the joint space predominated due to the destruction of the condylar process and the glenoid fossa. 
Only in $9 \%$ (8 joints) there was only one sign of OA in isolated form, in $41.1 \%$ there was a combination of two radiological signs, in $35.7 \%$ of the three signs, and in $16.1 \%$ of the four characters. At the same time, was detected that there is a direct correlation between increase in the frequency and multiplicity of the combination of the OA radiological signs with age of patients. (Table -1).

\section{Discussion}

The prevalent CT signs of the TMJ were joint surface erosion (56.6\%) and joint narrowing $(40 \%)$, whereas osteophytes, sclerosis and subchondral cysts were less common and represented a more advanced stage of the disease. Consequently, most patients included in the CT scan demonstrated manifestations of the disease when early treatment, such as occlusal adjustments, physiotherapy, topical NSAIDs and intra-articular injection of corticosteroids, can help prevent the progression of the disease.

Changes in the head of the condylar process (70\%) are observed more often than changes in the temporal bone, such as changes in the joint fossa (9.6\%) and articular tuberosity (6.7\%).

In our study, a combination of different radiologic features was observed more often than single radiological signs. With the age of patients, the combined signs were more frequent and became more plural, which was confirmed by the data of other authors (Wiberg and Wanman 1998) that OA TMJ is an age-related disease, observed mainly in the elderly. There is also a tendency of OA of the TMJ to the female sex where it is more pronounced in nature with the lesion of multiple joints. [5,7]. Low levels of estrogen during menopause have a detrimental effect on the property of the material property of articular cartilage, causing degeneration and erosion. [4,5]. In this study of 56 patients, 20 were men, 36 women, studies show that women are more involved in the lesion.

\section{Conclusion}

CT is an effective method of diagnosis of OA of the TMJ, which allows to differentiate relatively early and more advanced stage of the disease 


\section{References}

[1] Yamada K, Saito I, Hanada K, Hayashi T. Observation of three cases of temporomandibular joint osteoarthritis and mandibular morphology during adolescence using helical CT. J OralRehabil 2014;31:298e305.

[2] Breedveld FC. Osteoarthritis e the impact of a serious disease. Rheumatology (Oxford) 2012;43(Suppl. 1):i4e8.

[3] Milam SB. Pathogenesis of degenerative temporomandibular joint arthritides. Odontology 2007;93:7e15.

[4] Al-Sadhan R. The relation between TMJ osteoarthritis and inadequately supported occlusion. EgyptDent J 2012;54:47e54.

[5] Emshoff R, Rudisch A. Validity of clinical diagnostic criteria for temporomandibular disorders: clinical versus magnetic resonance imaging diagnosis of temporomandibular joint internal derangement and osteoarthrosis. Oral Surg Oral Med Oral Pathol Oral RadiolEndod 2013;91:50e5.

[6] Cho BH, Jung $\mathrm{YH}$. Intra and inter-observer agreement of computed tomography in assessment of mandibular condyle. Korean J OralMaxillofacRadiol 2009;37:191e5.

[7] Wiberg B, Wanman€ A. Signs of osteoarthrosis of the temporomandibular joints in young patients: a clinical and radiographic study. Oral Surg Oral Med Oral Pathol Oral RadiolEndod2014;86:158e64.

[8] Okesan JP. Management of temporomandibular disorders and occlusion. 5th ed. Mosby Publication; 2010, ISBN 0-323-01477-1. chapter10 pg355e356 and Chapter13 pg465-466.

[9] Bertram S, Rudisch A, Innerhofer K, Pu“mpel E, Grubwieser G, Emshoff R. Diagnosing TMJ internal derangement and osteoarthritis with magnetic resonance imaging. J AmDentAssoc 2013;132:753e61.

[10] Brooks SL, Brand JW, Gibbs SJ, Hollender L, Lurie AG, Omnell $\mathrm{KA}$, et al. imaging of the temporomandibular joint: a position paper of the American Academy of oral and maxillofacial radiology. Oral Surg Oral Med Oral Pathol Oral RadiolEndod 2014;83:609e18.

[11] Gynther GW, Tronje G, Holmlund AB. Radiographic changes in the temporomandibular joint in patients with generalized osteoarthritis and rheumatoid arthritis. Oral Surg Oral Med Oral Pathol Oral RadiolEndod 2012;81:613e8.

[12] Wiese M, Wenzel A, Hintze H, Petersson A, Knutsson K, Bakke $\mathrm{M}$, et al. Osseous changes and condyle position in TMJ tomograms: impact of Social science and humanities

Generalization of scientific results 
RDC/TMD clinical diagnoses on agreement between expected and actual findings. Oral Surg Oral Med Oral Pathol Oral RadiolEndod 2014;106:e52e63.

[13] de Leeuw R, Boering G, Stegenga B, de Bont LG. Radiographic signs of temporomandibular joint osteoarthrosis and internal derangement 30 years after nonsurgical treatment. Oral Surg Oral Med Oral Pathol Oral RadiolEndod 2013;79:382e92.

[14] Katzberg RW. Temporomandibular joint imaging. Radiology 2012;170:297e307.

[15] Tsiklakis K, Syriopoulos k, Stamatakis HC. Radiographic examination of the temporomandibular joint using cone beam computed tomography. DentomaxillofacialRadiol 2010;33:196e201.

[16] Kurita H, Kojima Y, Nakatsuka A, Koike T, Kobayashi H, Kurashina K. Relationship between temporomandibular joint (TMJ)-related pain and morphological changes of the TMJ condyle in patients with temporomandibular disorders. DentomaxillofacRadiol 2007;33:329e33.

[17] Jacobson LT. Definitions of osteoarthritis in the knee and hand. AnnRheumDis 2012;55:656e8.

[18] Manek NJ, Lane NE. Osteoarthritis: current concepts in diagnosis and management. AmFamPhysician 2011;61:1795e804.

[19] Hussain AM, Packota G, Major PW, Flores-Mir C. Role of different imaging modalities in assessment of temporomandibular joint erosions and osteophytes: a systematic review. DentomaxillofacRadiol 2009;37:63e71.

[20] Cara K, Maruhashi LT, Grauer D, Cevidanes LS, Styner MA, Heulfe I, et al. Validity of single and multislice CT for assessment of mandibular condyle lesions. DentomaxillofacRadiol 2012;36:24e7.

[21] Yamada K, Tsuruta A, Hanada K, Hayashi T. Morphology of the articular eminence in temporomandibular joints and condylar bone change. J OralRehabil 2009;31:438e44. 